\title{
Estudio sobre habilidades profesionales del ingeniero en zootecnia con enfoque en la educación física: Caso Ecuador
}

\author{
Study on professional skills of the engineer in zootechnics with a focus on \\ physical education: Case Ecuador
}

Fausto Iván Guapi Guamán ${ }^{1}$, Nelson Rene Ortiz Naveda², Manfredy Ismael Chugñay Cargua $^{3}$

\begin{abstract}
The mission of the polytechnic schools and university of Ecuador is to train competitive professionals, entrepreneurs, with values and principles, identified with the local, regional and national reality. That is the starting point and link between the academy and the productive and social sectors of the country that justifies the importance of generating research that guides and guarantees the integral training of the Engineers in Zootechnics. This work has the objective of carrying out the study on the development of the professional skills of the Engineer in Zootechnics with a focus on the subject of Physical Education in Ecuador, for this a study was made in the provinces of Orellana and Napo. The population was made up of professionals who work in both public and private companies. The results demonstrate the need to redesign the career project according to the need of the professional with an agricultural profile and therefore the academy within its competence must restructure the basic contents and teaching-learning methodologies that are executed in the careers of Zootechnics and orient them towards the needs that are needed according to the broad, specific and detailed field.
\end{abstract}

Keywords: Ecuador, physical education, profesional skills, zootechnics

\footnotetext{
${ }^{1}$ Escuela Superior Politécnica de Chimborazo, Extensión Norte Amazónica, Ingeniería en Zootecnia, Francisco de Orellana, Ecuador, fausto.guapi@espoch.edu.ec

2 Escuela Superior Politécnica de Chimborazo, Extensión Norte Amazónica, Ingeniería en Zootecnia, Francisco de Orellana, nelson.ortiz@espoch.edu.ec

${ }^{3}$ Escuela Superior Politécnica de Chimborazo, Extensión Norte Amazónica, Ingeniería en Zootecnia, Francisco de Orellana, Ecuador, machugñay@espoch.edu.ec
} 


\section{Resumen}

La misión que tienen las escuelas politécnicas y universidad del Ecuador es formar profesionales competentes, emprendedores, con valores y principios, identificados con la realidad local, regional y nacional. Ese es el punto de partida y de vinculación de la academia con los sectores productivos y sociales del país que justifica la importancia de generar investigación que oriente y garantice la formación integral de los Ingenieros en Zootecnia. Este trabajo tiene el objetivo de realizar el estudio sobre el desarrollo de las

habilidades profesionales del Ingeniero en Zootecnia con enfoque en la asignatura de Educación Física en el Ecuador, para ello se hizo un estudio en las provincias de Orellana y Napo. La población estuvo constituida por los profesionales que laboran tanto en empresas públicas como privadas. Los resultados demuestran la necesidad de rediseñar el proyecto de carrera de acuerdo a la necesidad labor del profesional con perfil agropecuario por lo tanto la academia dentro de su competencia debe reestructurar los contenidos básicos y metodologías de enseñanza- aprendizaje que se ejecutan en las carreras de Zootecnia y orientarlos hacia las necesidades que necesitan los profesionales de acuerdo al campo amplio, específico y detallado.

Palabras claves: Ecuador, educación física, habilidades profesionales, zootecnia

\section{Introducción}

La Educación Física es asignatura de gran importancia, ya que los conocimientos teóricos y prácticos que aporta dan al egresado herramientas para garantizar la calidad del proceso de enseñanza aprendizaje de la Educación Física posibilitando la incidencia de esta en las esferas social-afectiva, cognitiva y motriz en la práctica. (Fresneda, Herrera, A., \& Álvarez, H., 2013, pág. 1)

En Latino América, la Educación Física es una asignatura básica en las carreras de ingenierías y licenciaturas de escuelas politécnicas y universidades que tienen el objetivo de crear cultura hacia la práctica de un deporte determinado; de acuerdo a Ruiz, et al. (Como se citó en Florin, 2011) esta asignatura forma parte del proceso pedagógico y tiene como fin específico el desarrollo de la capacidad de rendimiento físico del individuo sobre la base del perfeccionamiento morfológico y funcional del organismo, la formación y mejoramiento de sus habilidades motrices, la adquisición de conocimientos y el desarrollo de convicciones, de forma tal que esté en condiciones de cumplir todas las tareas que la sociedad señale.

Entorno al tratamiento de la Educación Física en las instituciones de educación superior, existen aportes de autores nacionales e internacionales tales como Fresneda, Herrera y Álvarez (2013), proponen un programa integrador de Educación Física para el desarrollo de las habilidades profesionales específicas dirigido a la formación profesional con perfil agropecuario; Charchabal, Aguirre, \& Pérez (2003), proponen un modelo integral del proceso docente educativo de Educación Física para la carrera de Ingeniería de Minas; Rodríguez, Y. (2009), analiza el papel de la Educación Física en el desarrollo de los educandos de la Universidad de la Ciencia Informática, UCI; López, Morffi, \& Arencibia (2005), diseñan un programa de Educación Física para la enseñanza superior; Concha, 
Alarcón, Angulo, Ayona, \& Guerra, (2014) realizan un análisis de la Educación Física en el Ecuador y el mundo; Rodríguez, Ruiz, \& De La Osa (2016); Toledo, Guapi, Herrera, \& Ariza (2019), realizan el estudio de la aplicabilidad de los conocimientos de la asignatura de educación física en el campo laboral turístico y Guapi \& Aldas (2019), abordan la Educación Física como asignatura complementaria en carreras de ingeniería en la educación superior ecuatoriana.

En la actualidad, en la República del Ecuador la asignatura de Educación Física “....es la asignatura que de manera obligatoria se imparte en todos los niveles pre-primario, educación general básica y bachillerato como una actividad de formación física, cultural, y humanística de los estudiantes.” (Aldas \& Gutiérrez, H., 2014, pág. 1). En lo que respecta a la educación superior se ha podido evidenciar estudios sobre la Educación Física, Guapi \& Aldas (2019) abordan el estudio de manera general en las escuelas politécnicas y universidades; Toledo, Guapi, Herrera, \& Ariza, (2019) realizan el estudio de la aplicabilidad de la asignatura en el ámbito turístico sin embargo no se evidencia estudios sobre las habilidades profesionales que debe desarrollar el Ingeniero en Zootecnia con enfoque en la Educación Física.

Al realizar la revisión del plan de estudios de la carrera de Zootecnia de algunas universidades del país, la observación es la misma, no se desarrolla una formación orientada a las necesidades y habilidades profesionales para desenvolverse en el campo laboral agropecuario. Los contenidos del programa analítico y los temas desarrollados que se enseña en la asignatura de Educación Física son en su mayoría, capacidades físicas y elementos técnicos de disciplinas como el fútbol, baloncesto y natación.

A partir de lo antes planteado se determina que existen las siguientes contradicciones:

- Insuficiencias en el tratamiento de la formación del profesional, no se desarrollan las habilidades profesionales.

- No se corresponde el desarrollo de las habilidades con el desempeño laboral en el vínculo con las clases de educación física.

Por lo que se plantea como problema científico, no existe el estudio sobre habilidades profesionales del Ingeniero en Zootecnia con enfoque en la Educación Física. El objetivo del trabajo investigativo, es realizar el estudio sobre el desarrollo de las habilidades profesionales del Ingeniero en Zootecnia con enfoque en la asignatura de Educación Física.

Como hipótesis se plantea que el programa actual de Educación Física, que se está ofertando y ejecutando en las instituciones de educación superior en las provincias de Napo y Orellana, no aportan con el desarrollo de conocimientos, habilidades y destrezas que realmente necesita el profesional en sus actividades agropecuarias.

\section{Métodos}

Para desarrollar el presente estudio se utiliza como métodos de investigación la revisión bibliográfica, para realizar la revisión sistemática de documentos de carácter científico en torno a propuestas de programas de Educación Física en la educación superior. El 
análisis de los documentos, con la finalidad de conocer los fundamentos teóricos que sustentan las diferentes propuestas de la temática. El criterio de inclusión y exclusión, a fin de seleccionar las propuestas referente a los contenidos de Educación Física que se debe impartir en las carreras de ingeniería y licenciatura; por otra parte los artículos descartados fueron las propuestas entorno a contenidos dirigido a estudiantes de la carrera de Educación Física.

\section{Metodología}

La población objetivo del presente estudio lo constituye los Ingenieros en Zootecnia así como técnicos en el área que laboran en las instituciones tanto públicas como privadas de la provincia de Napo y Orellana; conformado por ochenta y cinco profesionales relacionados en la rama de Zootecnia, la información fue obtenida directamente mediante una investigación de campo empleando la técnica de encuesta. Se establecieron la dimensión habilidades profesionales del Ingeniero en Zootecnia propuesto por Fresneda, Herrera, A., \& Álvarez, H., (2013) y la dimensión campo laboral (amplio y específico) del Ingeniero en Zootecnia. Para el efecto se determinó la muestra considerando los datos que se evidencian a continuación:

Fórmula aplicada para el cálculo de la muestra

- $\mathrm{Z}^{2}$. N. p.q

- $\mathrm{n}=$

- $\mathrm{i}^{2}(\mathrm{~N}-1)+\mathrm{Z}^{2} \cdot \mathrm{p} \cdot \mathrm{q}$

- (N) Tamaño del universo: 85 profesionales.

- Error: $5 \%$

- (z) Nivel de Confianza 90\% $(1,65)$

- (p) Probabilidad de ocurrencia $50 \%$

- Tamaño de la muestra 68 profesionales.

El instrumento para la encuesta fue un cuestionario que fue previamente validado por un equipo de profesionales de la carrera de Zootecnia de la Escuela Superior Politécnica de Chimborazo Extensión Norte Amazónica. La encuesta se la aplicó en línea utilizando la herramienta disponible en google drive con un tipo de muestreo probabilístico - aleatorio simple (muestreo simple al azar).

\section{Resultados}

En la Republicad del Ecuador la Educación Física a nivel superior se alinea de acuerdo a lo que manifiesta la Ley del Deporte, Educación Física y Recreación, en vigencia desde el 10 de agosto de 2010, en la décimo octava disposición general; que plantea: "Educación Física es una disciplina que basa su accionar en la enseñanza y perfeccionamiento de movimientos corporales, busca formar de una manera integral y armónica al ser humano, estimulando positivamente sus capacidades físicas." (Asamblea Nacional, 2010, pág. 24)

En apego a lo que manifiesta la Ley del Deporte Educación Física y Recreación las universidades y escuelas politécnicas del Ecuador, tienen como objetivo desarrollar y mejorar las cualidades físicas, técnicas, tácticas, psicológicas y volitivas de los 
estudiantes a través de la práctica de un determinado deporte; por lo que se limitan únicamente a la práctica de fútbol, básquet, atletismo, entre otros, sin embargo a través de las clases de educación física se puede desarrollar habilidades profesionales de cada profesión y de manera específica del egresado del Ingeniero en Zootecnia. De acuerdo con lo que plantea Fresneda et al. (2013), la Educación Física en la educación superior aporta al egresado herramientas que garantizan la calidad del proceso de enseñanzaaprendizaje de la asignatura, incidiendo en las esferas social-afectiva, cognitiva y motriz que se promueven en las clases de Educación Física.

Por otra parte, el Centro de Educación Física, Deportes y Recreación de la Escuela Superior Politécnica de Chimborazo, de acuerdo con el Estatuto Politécnico tiene la misión de “... apoyar en la formación integral de los estudiantes politécnicos en el área de la educación física, masificar y fomentar la práctica del deporte y la recreación." (Escuela Superior Politécnica de Chimborazo Secretaria General, 2004, pág. 35).

A decir de la formación integral en el nivel superior no solo debe centrarse en la formación de profesionales competentes, sino además en formar profesionales éticos, comprometidos a promover la paz, la defensa de los derechos humanos y los valores de la democracia. En concordancia con lo que plantean Guerra, Mórtigo, \& Berdugo, (2013), manifiestan:

Es una forma de brindar la educación, un estilo educativo que pretende, no solo instruir a los estudiantes en su aspecto mental, memorístico, repetitivo, con los saberes específicos (aspecto cognitivo) de las ciencias; sino, también, ofrecerles los elementos necesarios para que crezcan como personas, buscando desarrollar todas sus características, condiciones y potencialidades. (pág. 52)

En este sentido la Educación Física como asignatura complementaria en la carrera de Zootecnia contribuye a la formación integral del estudiante, en primera instancia mediante los contenidos que se imparte promueve los saberes específicos de la asignatura; por otra parte, trabaja los aspectos de la persona de acuerdo con lo que plantean Fresneda, Herrera, A., \& Álvarez, H. (2013).

(...) su práctica se impulsa los movimientos creativos e intencionales, la manifestación de la corporeidad a través de procesos afectivos y cognitivos de orden superior. De igual manera, se promueve el disfrute de la movilización corporal y se fomenta la participación en actividades caracterizadas por cometidos motrices. (pág. 1)

El Ingeniero en Zootecnia según el Report de carrera posee campo amplio de acción declarado como agricultura, silvicultura, pesca y veterinaria donde podrá ocupar puesto de trabajo en empresas públicas y privadas, en las que cumple las siguientes misiones:

- Asesoría y manejo del crecimiento pecuario local, regional y nacional en entidades públicas y privadas.

- Aplicación de métodos para producir y transformar eficientemente los alimentos de origen animal, utilizando procedimientos modernos de cría, alimentación, mejoramiento y manejo de rebaños y plantas. 
- Elaborar, diseñar y ejecutar, programas, proyectos, encaminados a asegurar la producción animal de forma eficiente.

- Aplicación de métodos para producir y transformar eficientemente los alimentos de origen animal, utilizando procedimientos modernos de cría, alimentación, mejoramiento y manejo de rebaños y plantas.

- Elaborar, diseñar y ejecutar, programas, proyectos, encaminados a asegurar la producción animal de forma eficiente.

- Administrar unidades productivas pecuarias propias y/o privadas.

- Ofrecer asistencia técnica especializada, sobre producción, manejo de ganado, alimentación, sanidad, mejoramiento y cría de las especies de interés zootécnico, así como de la producción de pastos y forrajes.

Ahora bien, con el fin de dar continuidad al presente artículo se plantea la siguiente interrogante ¿Cuáles son las habilidades profesionales del Ingeniero en Zootecnia que se debe desarrolla a través de la asignatura de Educación Física?; para responder el planteamiento anterior se debe empezar hablando sobre lo que es la habilidad. La habilidad ha sido abordada desde dos posturas de la psicología y la pedagógica actual, desde la psicología se la considera como el "(...) dominio de un complejo sistema de acciones psíquicas y prácticas necesarias para una regulación racional de la actividad, con ayuda de los conocimientos y hábitos que la persona posee" (Petrovsky, 1985, pág. 159).

Para que exista la formación de una habilidad como bien se refiere en el concepto anterior debe existir el dominio de un complejo sistema de acciones y además operaciones, sin entrar en detalle sobre las acciones y operaciones puesto que no es el objetivo del artículo se plantea que, tanto la acción como la operación no constituyen de ningún modo algo "separado" de la actividad (Rodríguez \& Bermúdez, R., 2004). Para realizar cualquier actividad es necesario tener en cuenta que la ejecución de la misma necesita de componentes ejecutores e inductores como bien plantea Montes de Oca \& Machado, R., (2009):

Cualquier forma de realización de una actividad, precisa de componentes ejecutores e inductores. De esta manera, la actividad se realiza a través de acciones y operaciones que constituyen los componentes ejecutores de la actividad; así la actividad está condicionada por los motivos, las acciones por los objetivos y las operaciones por las condiciones. (pág. 7)

En este sentido, toda actividad humana se caracteriza por la motivación que es inducida y se materializa a través de las acciones y las operaciones "El carácter consciente de la acción está en la complejidad de su ejecución.” (Rodríguez,M., 2004, pág. 6). Pero en este proceso de ejecución de la actividad de manera conjunta se encuentra la operación la cual se puede desarrollar de manera inconciente. De esta afirmación se infiere que: "Toda acción es consciente como toda operación es inconsciente" (Rodríguez,M., 2004, pág. 9). En este sentido los contenidos que imparte el docente al estudiante deben tributar el desarrollo a nivel tanto subjetivo como práctico.

De lo anterior se infiere que para desarrollar una determinada habilidad debe generarse acciones las mismas que variaran en dependencia a la habilidad a trabajar; ahora bien, 
para determinar las acciones que componen la habilidad a desarrollar en el Ingeniero en Zootecnia; se debe considerar el medio laboral que se va a desenvolver el futuro profesional.

Dentro de la clasificación de habilidades de acuerdo con Rodríguez \& Bermúdez, (1993) se clasifican en teóricas y prácticas; por un lado, la habilidad es práctica cuando se pueden seguir todas las partes del proceso de actividad hasta su resultado, por estar presente el componente motor. Y es teórica cuando sólo puede controlarse el resultado final, pues las distintas acciones que componen dicha habilidad transcurren en el plano interno mental.

Ahora bien, la base sobre la cual se forma y desarrolla una habilidad tanto teórica como práctica es el conocimiento y los hábitos es decir el estudiante a través del conocimiento impartido por el docente y la constante practica o puesta en práctica de un tema en la clase de educación física contribuirá a la formación de la habilidad. De este hecho también se infiere la razón por lo cual la clase de educación física debe contener la parte teórica como práctica.

Antes de finalizar el apartado de habilidades es necesario abordar sobre el termino capacidad, en algunos casos se cree que son iguales; sin embargo, al referirnos a capacidad se hace referencia a las particularidades psíquicas particulares que posee cada uno y hacen único a las personas. "El hecho de poseer determinados conocimientos, hábitos y habilidades no implica tener desarrollada una capacidad, si bien facilita su formación; es sólo mediante la experiencia práctica de varios años que un individuo puede llegar a ser verdaderamente capaz.” (Rodríguez \& Bermúdez, 1993, pág. 31).

Para que las capacidades propias de su actividad se desarrollen de manera óptima durante el tiempo de desempeño laboral el futuro Ingeniero en Zootecnia debe poseer aptitudes y adecuada motivación interna para ejercer las actividades de su puesto laboral.

Las denominadas habilidades profesionales son desarrolladas fundamentalmente desde disciplinas y asignaturas del ejercicio de la profesión; no obstante, las del ciclo básico o básico específico de alguna manera deben tributar también a la formación y desarrollo de dichas habilidades de modo que sea posible desde ellas, crear condiciones para su desarrollo. Ello puede lograrse si se tiene en cuenta el entramado de relaciones entre las específicas y las profesionales al planificar el proceso, enfatizando en las integradoras y esenciales que sean determinantes para el desarrollo de otras, así como en los procesos y sus relaciones.

Por consiguiente la Educación Física posee un papel integrador, por el vínculo que tiene con otras asignaturas de la carrera y no limitándose únicamente al perfeccionamiento o desarrollo de la condición física del profesional a través del ejercicio sino además coadyuva al desarrollo integral incidiendo en la esfera social-afectiva mediante los juegos o ejercicios donde promueva la cohesión del grupo, valores y en la esfera cognitiva mediante juegos de razonamiento se trabaja la parte intelectual del alumno; además mediante esta asignatura se puede desarrollar la preparación específica y habilidades profesionales específicas necesarias del futuro Ingeniero en Zootecnia. 
Para el estudio de la presente investigación se asume los contenidos para desarrollar habilidades profesionales en las clases de educación física planteadas por Fresneda, Herrera, A., \& Álvarez, H., (2013). Los cuales se encuentran relacionados con:

- Caballería (nudos y amarres).

- Estimación de distancia y altura.

- Natación y salvamento.

- Equitación (preparación y monta de caballo).

- Orientación en el terreno.

Con el objetivo de conocer las habilidades profesionales, el campo ocupacional, el desempeño profesional y campo de intervención de los Ingenieros en Zootecnia se aplicó encuestas a los profesionales que laboran en empresas públicas y privadas correspondientes a la Zona 2 (Orellana y Napo) excluyendo a la provincia de Pichincha. $\mathrm{Y}$ al ser tabulado los instrumentos aplicados se obtuvo los resultados detallados a continuación.

Figura 1. Contenidos y técnicas a desarrollar en las clases de Educación Física

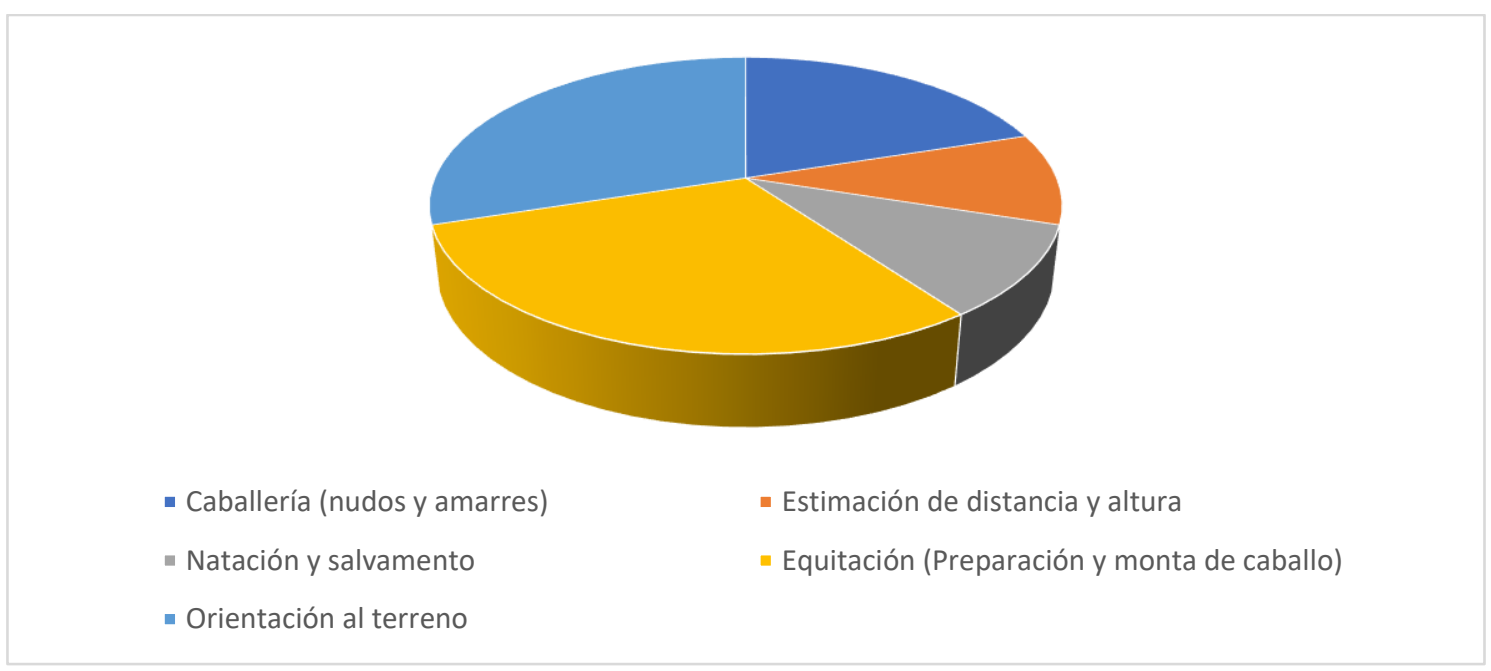

Fuente: Elaboración propia de los autores

El criterio respecto a los contenidos y técnicas que se deberían desarrollar en la asignatura de Educación Física para desempeñarse como profesionales en el área agropecuaria recae en caballería (nudos y amarres) con un 30\%; seguidos por la equitación (preparación y monta de caballo) con el 25\%; posteriormente están los conocimientos para orientación al terreno con $20 \%$ y como últimos se encuentran natación y salvamento, estimación de distancia y altura con el $14 \%$ y el $11 \%$ respectivamente. 
Figura 2. Campo ocupacional de los Ingenieros en Zootecnia

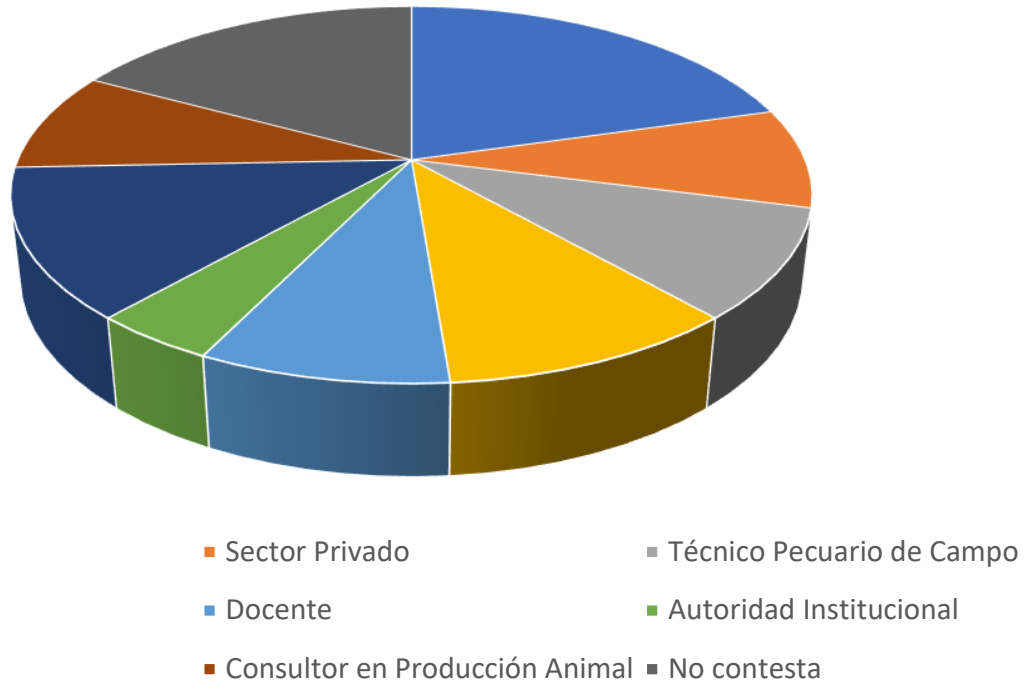

Fuente: Elaboración propia de los autores

El 20,05\% se encuentra laborando en el sector público, seguido del 10,05\% que trabaja en el sector privado. El 11,25\% se desempeña como técnico pecuario de campo; $12,46 \%$ como administrador; $9,05 \%$ como docente; $6,02 \%$ como autoridad institucional (IES); $14,07 \%$ asesor pecuario; $12,05 \%$ consultor en producción animal y el 5,0\% se abstiene en contestar la pregunta de la encuesta.

Figura 3. Ámbitos de desempeño profesional de los Ingenieros en Zootecnia

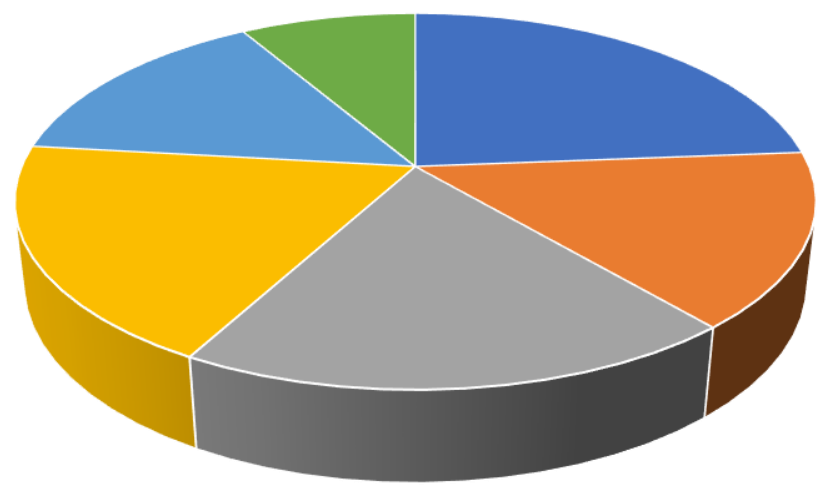
- Reproducción Animal
- Genética y Mejoramiento Ganadero
- Desarrollo Sustentable
- Salud Animal
- Administración Pecuaria
- Nutrición Animal

Fuente: Elaboración propia de los autores 
En base a los campos de intervención del profesional en Zootecnia en los cuales se desempeña, a continuación, se detalla los porcentajes: 23,75\% en Reproducción Animal, acompañado del 15,02\% de Genética y Mejoramiento Ganadero; Desarrollo Sustentable el 19,19\%; 18,85\% en Salud Animal; 14,72\% en Administración Pecuaria y el 8,47\% en Nutrición Animal.

Figura 4. Campos de intervención de los profesionales en Zootecnia

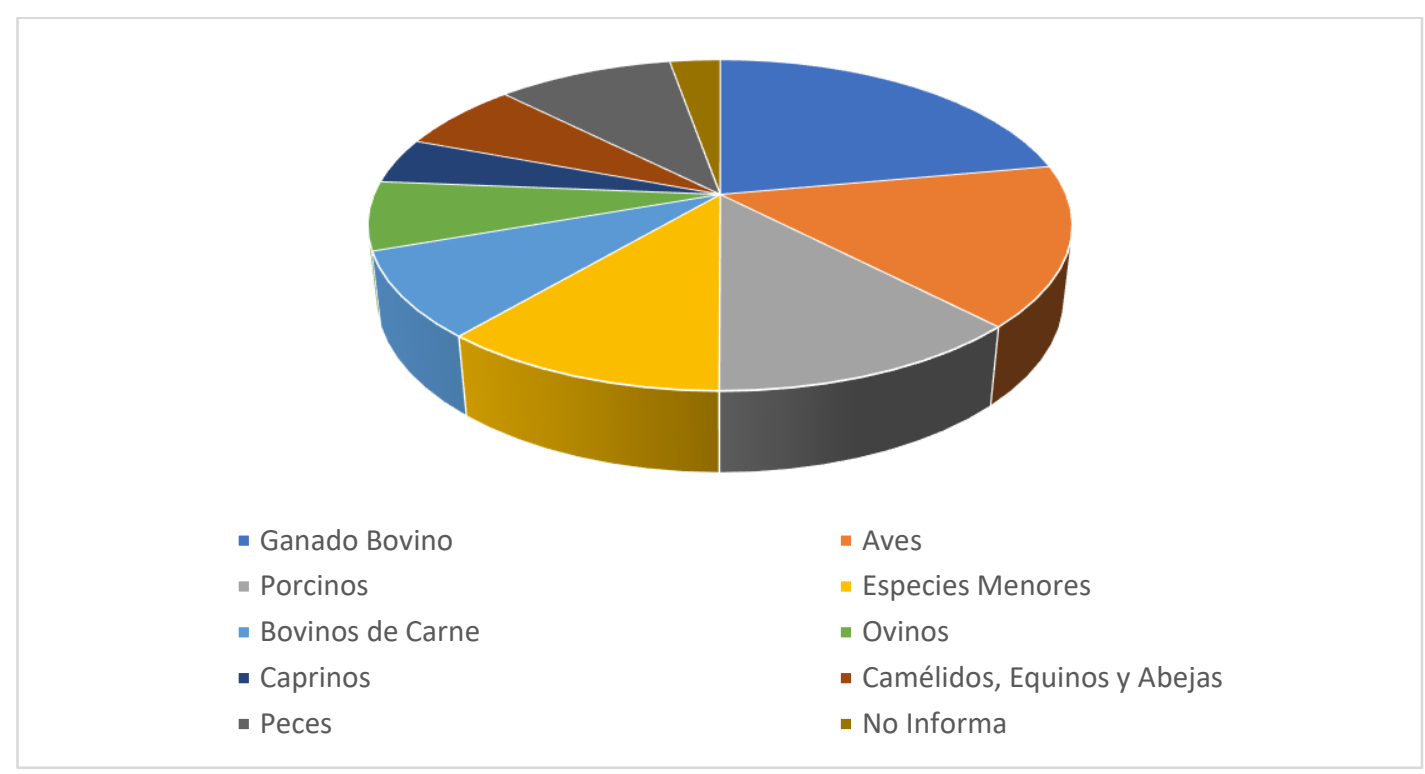

Fuente: Elaboración propia de los autores

Cabe indicar que los semovientes que se dedican a explotar los profesionales zootecnistas son: ganado bovino con el $22,11 \%$; seguido de las aves con el 15,64\%; porcinos $12,29 \%$; especies menores $11,17 \%$; bovinos de carne $8,38 \%$; ovinos $6,70 \%$; caprinos $4,47 \%$; y camélidos, equinos y abejas $6,79 \%, 1,68 \%$ peces respectivamente y el $2,77 \%$ no informa.

Las figuras anteriores muestran evidencias en primer instancia sobre los contenidos que se debería impartir en las clases de educación física y en segunda instancia sobre el campo amplio, campo específico y campo detallado donde hasta el momento los Ingenieros en Zootecnia se encuentran laborando y con estos resultados proponer un programa analítico para la asignatura de Educación Física con los contenidos que contribuyan al desarrollo de habilidades profesionales en la carrera de Zootecnia.

\section{Conclusiones}

- La asignatura de Educación Física como asignatura básica dentro de la malla del Ingeniero en Zootecnia contribuye a desarrollar habilidades profesionales propias del profesional con perfil agropecuario. Sin embargo al no existir estudios de carácter técnico que puedan sustentar la imperiosa necesidad de rediseñar los contenidos de la asignatura se continuará limitando a la enseñanza de los elementos básicos de los deportes.

- Los resultados arrojados de la presente investigación demuestran que existe interés por los profesionales que se integren los contenidos en proyecto de carrera y por consiguiente en el programa analítico en la asignatura de Educación Física ubicándose con mayor puntaje caballería (nudos y amarres) y la equitación (preparación y monta 
de caballo) con el $30 \%$ y $25 \%$ respectivamente. Además se debe tener en cuenta que el mayor número de profesionales se encuentran laborando en campo distribuido de la siguiente manera 23,75\% en Reproducción Animal, acompañado del 15,02\% de Genética y Mejoramiento Ganadero; Desarrollo Sustentable el 19,19\%; 18,85\% en Salud Animal; 14,72\% en Administración Pecuaria y el 8,47\% en Nutrición Animal.

\section{Referencias bibliográficas}

Aldas, G., \& Gutiérrez, H. (2014). La Cultura Física y su formación profesional en la República del Ecuador. EFDeportes.com, Revista Digital, 19(199), 1-1. Obtenido de http://www.efdeportes.com/efd199/la-cultura-fisica-y-su-formacion-enecuador.htm

Asamblea Nacional. (2010). Ley del Deporte, Educación Física y Recreación. 24. Ecuador.

Charchabal, D., Aguirre, A., \& Pérez, M. (2003). Elaboración del modelo integral del proceso docente educativo de Educación Física para la carrera de Ingeniería de Minas. EFDeportes.com, Revista Digital, 9(65), 1-2. Obtenido de https://www.efdeportes.com/efd65/minas.htm.

Concha, C., Alarcón, G., Angulo, C., Ayona, A., \& Guerra, J. (2014). Breve análisis de la Educación Física en el Ecuador y el mundo. EFDeportes.com, Revista Digital, 19(196). Obtenido de https://www.efdeportes.com/efd196/la-educacion-fisicaen-el-ecuador.htm.

Fresneda, C., Herrera, A., \& Álvarez, H. (2013). La Educación Física universitaria: una actualidad con perspectiva. EFDeportes.com, REvista Digital, 17(178), 1-1. Obtenido de http://www.efdeportes.com/efd178/la-educación-fisicauniversitaria-una-actualidad.htm

Guapi, F., \& Aldas, H. (2019). La Educación Física como asignatura complementaria en carreras de ingeniería en la Educación Superior Ecuatoriana. 14(1), 93-106. Obtenido de http://podium.upr.edu.cu/index.php/podium/article/view/784.

Guerra, Y., Mórtigo, A., \& Berdugo, N. (2013). Formación integral importancia de formar pensando en todas las dimensiones del ser. Educación y Desarrollo Social, 8(1), 48-69. Obtenido de https://dialnet.unirioja.es/descarga/articulo/5386176.pdf.

López, H., Morffi, A., \& Arencibia, R. (2005). Diseño de un programa de Educación Física para la enseñanza superior. EFDeportes.com, Revista Digital, 10(90), 1-1. Obtenido de https://www.efdeportes.com/efd90/superior.htm

Montes de Oca, N., \& Machado, R. (2009). El desarrollo de habilidades investigativas en la educación superior: un acercamiento para su desarrollo. Humanidades Médicas, 9(1), 4-15. Obtenido de http://scielo.sld.cu/scielo.php?script=sciarttext\&pid=S1727$81202009000100003 \& \operatorname{lng}=$ es\&tlng=es

Petrovsky, A. (1985). Psicología general. Moscú: Editorial Progreso. 
Rodríguez, F., Ruiz, F., \& De La Osa, O. (2016). La Educación Física en el Plan de estudio "E" de la Educación Superior Cubana. 1(2), 19-35. Obtenido de https://revistas.reduc.edu.cu/index.php/cienciaydeporte/artcle/view/1668

Rodríguez, M., \& Bermúdez, R. (1993). Algunas consideraciones acerca del estudio de las habilidades. Revista Cubana de Psicología, 10(1), 27-32. Obtenido de http://pepsic.bvsalud.org/pdf/rcp/v10n1/04.pdf.

Rodríguez, M., \& Bermúdez, R. (2004). Habilidades profesionales, ¡no! Hábitos profesionales, isi!. Obtenido de http://www.sld.cu/galerias/doc/sitios/prevemi/habilidades_profesionalesno.doc.

Rodríguez, Y. (2009). El papel de la Educación Física en el desarrollo de los educandos de la Universidad de Ciencias Informática, UCI. EFDEPORTES.com, Revista Digital, 13(130), 1-1. Obtenido de https://www.efdeportes.com/efd130/laeducacion-fisica-la-universidad-de-la-ciencia-informatica.htm.

Toledo, M., Guapi, F., Herrera, R., \& Ariza, A. (2019). Estudio de la aplicabilidad de los conocimientos de la Educación Física en el campo laboral turístico: caso Ecuador. Polo del Conocimiento, 4(2), 266-278. doi:10.23857 


\section{Para citar el artículo indexado.}

Guapi Guamán, F., Ortiz Naveda, N. R., \& Chugñay Cargua, M. (2019). Estudio sobre habilidades profesionales del ingeniero en zootecnia con enfoque en la educación física: Caso Ecuador. Explorador Digital, 3(3), 77-89. https://doi.org/10.33262/exploradordigital.v3i3.752

\section{$\lfloor$ Ciencia}

El artículo que se publica es de exclusiva responsabilidad de los autores y no necesariamente reflejan el pensamiento de la Revista Explorador Digital.

El articulo queda en propiedad de la revista y, por tanto, su publicación parcial y/o total en otro medio tiene que ser autorizado por el director o editor de la Revista Explorador Digital.
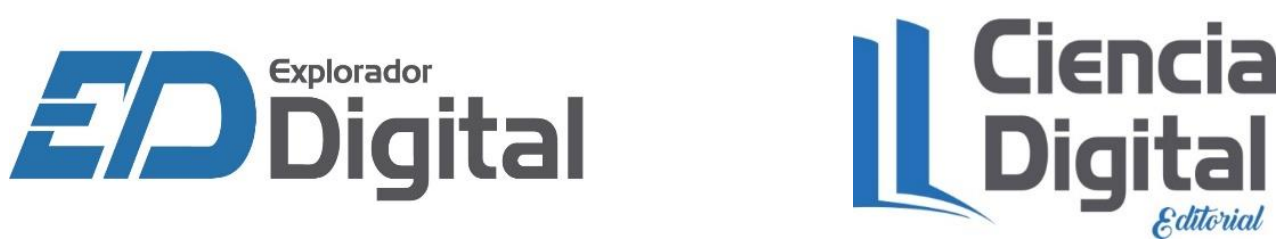